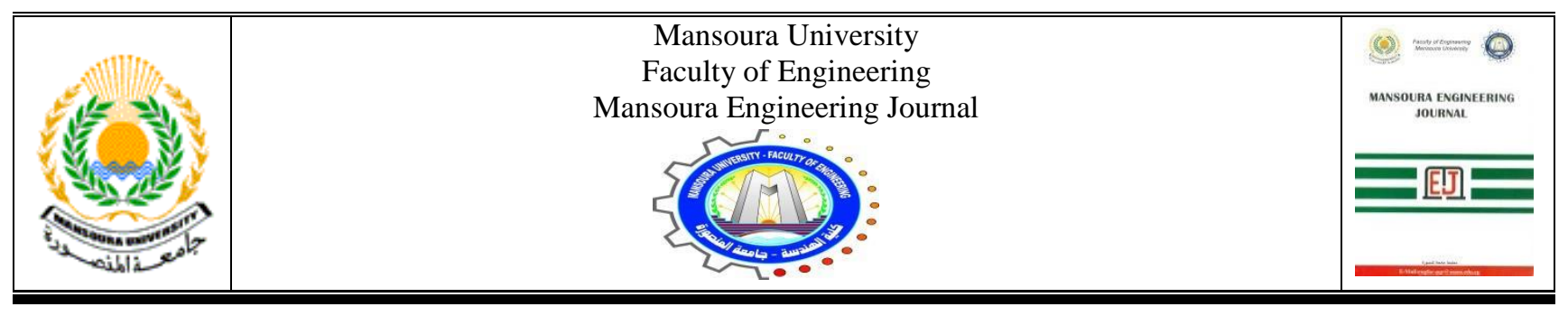

\title{
Modeling of Conjugate Heat Transfer
}

\author{
نمذجة انتقال الحرارة المركب
}

\author{
Mohamed R. Elmarghany, Mohamed H. Mansour, Ahmed A. Sultan and Mohamed Nabil Sabry. \\ Mechanical Power Engineering Department, Faculty of Engineering, \\ Mansoura University, El-Mansoura 35516, Egypt \\ Emails:Mohamed_ragab@mans.edu.eg,mhsaadanym@mans.edu.eg,mnabil.sabry@gmail.com
}

\begin{abstract}
KEYWORDS
Conjugate Heat transfer -

Compact Thermal model -

Heat Transfer Coefficient-

laminar heat transfer
\end{abstract}

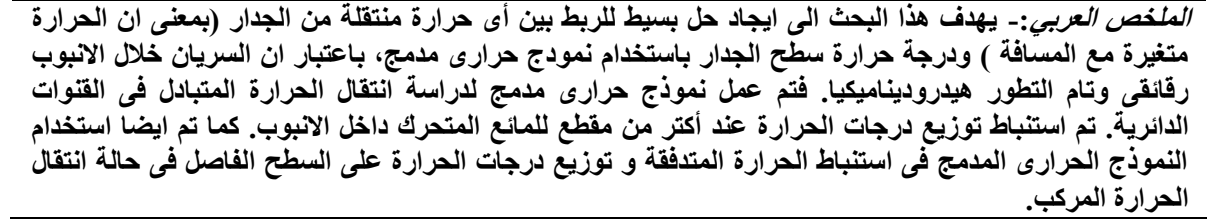

\begin{abstract}
Conjugate heat transfer in ducts is one of the most popular research topics due to its wide engineering applications. Most of the interest on the heat transfer problems is the two cases of the uniform wall temperature and uniform heat flux. However, the heat-transfer problems with non-uniform heating are also investigated by a few studies. The purpose of this work is to obtain a more general simple expression for any heat flux density and temperature profiles using compact thermal model which offers many advantages over the traditional approach using the heat transfer coefficient (i.e. any space distribution, not just uniform). Thus, a steady state compact thermal model is established and used to study Conjugate heat transfer through circular duct. The flow is considered to be laminar and hydrodynamically fully developed through the entire duct length. The temperature distributions of the moving fluid inside the duct at different sections have been presented for all investigated cases. The compact thermal model also used to investigate temperature distribution and the heat flux at the interface for conjugate heat transfer.
\end{abstract}

\section{INTRODUCTION}

$\mathrm{C}$ onjugate heat transfer, which is the ultimate goal in this work, is a coupled problem involving heat transfer in a fluid domain as well as in the surrounding solid wall. Heat transfer by conduction and convection simultaneously occurs in the majority of engineering applications, such as heat exchangers and fins. Efficiently combining heat transfer in solids and fluids is the key to design effective coolers, heaters, or heat exchangers.

Modeling and design of such heat transfer devices is complicated due to coupling, which involves a rather large number of parameters describing both fluid and solid domains. An intelligent strategy is to decouple the problem by modeling each domain alone, followed by merging models of both domains using continuity conditions at the solid/fluid interface, which are:

- Continuity of temperature field

- Continuity of heat flux

Each model should describe the relation between heat fluxes and temperature differences across the domain. It must be a rather simple model, compared to the complete description involving the solution of the energy partial differential equation (PDE). Yet, it must also be accurate enough to take into consideration the variation of temperature and heat flux fields along the solid / fluid interface.

Above requirements lead to the creation of intermediate level models, called Compact thermal model (CTM), which lie between the over simplified level of the heat transfer coefficient (HTC) on one side and the complex PDE level. This will be done in this work for the classical straight circular duct numerically. Obtained models will be merged to solve the conjugate problem.

Many numerical studies were done to study conjugate effect in circular and noncircular ducts. Campo and Schuler [1] examined the influence of a finite heated length on the heat transfer characteristics of fully developed laminar flow through circular ducts with thick walls. This kind of conjugate problem was governed by four dimensionless groups: the Peclet number, the solid-fluid thermal conductivity ratio, the length of the heated region, and the diameters ratio of the solid wall. From their numerical results, it was found that the two-dimensional wall offers a heat flow pathway into fluid flow controlling the parameters of interest such as, the bulk temperature of 
fluid and both external and internal surface temperatures of the solid wall. From a set of typical cases analyzed, it was concluded that both surface temperatures showed an essential variation in the axial direction and lesser and more gradual variations were shown for the distribution of bulk temperature. A numerical study for laminar conjugate heat transfer in a tube subjected to an axially non-uniform heat flux at the outer surface of the tube has been investigated by Aydin et al. [2] using the finite volume approach. The effects of the diameter ratio and the thermal conductivity ratio as well as the temperature and the interface heat flux distribution are determined for a range of corresponding parameters. Luna et al. [3] presented the steady-state analysis of conjugate heat transfer for the thermal entrance region for developed laminar flow forced convection in a circular duct. A uniform heat flux was applied at the external surface of the duct. The energy equation was solved analytically by using the integral boundary layer approximation, neglecting the heat generation due to viscous dissipation and the axial heat conduction in the fluid. Furthermore, the analysis of the thermal stresses in fully developed laminar flow was presented by AlZaharnah et al. [4, 5]. They applied a uniform heat flux at the outer surface of the duct and solved the governing energy equation numerically by using a control volume approach. Barozzi and Pagliarini [6] proposed a general procedure combining the superposition principle with a finite element method to solve conjugate heat transfer problems. This method considered the wall conduction effect on heat transfer of fully developed laminar flow through a circular duct with uniform heat flux. Shah and Jain [7] developed an iterative method to solve conjugate heat transfer problems analytically. According to an initial assumption of the temperature field at the fluid-solid interface, the temperature distributions in the solid domain and the fluid domain were determined by solving the governing energy equations in the solid and fluid domains separately. These solutions were used to iterate the initial assumption of the interface temperature until convergence. Luikov et al. [8] presented solution methods of convective heat transfer problems which considered heat diffusion in the solid in contact with a fluid. They discussed heat transfer in laminar fluid flow in circular and non-circular ducts. In addition, they considered both steady- and unsteady-state heat transfer problems for flow of a compressible fluid. In all introduced cases heat transfer in the fluid was discussed in relation to that in a solid.

Many of the previous works were made based on the traditional approach using HTC. This is too simple to dissect a large number of convective heat transfer problems found in reality. Hence, the CTM is considered to be widely used in modeling convection problems. A compact model $[9,10]$ defined as simple relation between heat fluxes and temperatures in an element, which involves a relatively small number of degrees of freedom. Many researches were made to improve the CTM approach. CTM have been constructed in electronic systems for different levels. Sabry [11] presented first steps toward constructing a unifying theory for linear systems giving general restrictions on the form of the compact model. The theory was used to study the completeness of the set of boundary conditions used to derive and validate CTMs.

From the previous review, it is found that most studies were made for standard cases (uniform $T$ or uniform $q$ ) based on Newton's law of cooling with its significant defects. Other studies were made numerically with 3D simulations which are more accurate, but consume more time. The CTM approach is used to bridge the gap between both approaches going gradually from simple HTC levels, to complicated 3D simulation. CTM will be established for forced convection heat transfer in circular ducts with non-uniform heat flux which is valid for any boundary conditions.

\subsection{Generalization of the Newton's Law of Cooling}

Convection is a complicated phenomenon that involves transfer of energy between an object and its environment, due to fluid stream near the object surface, which is not at the same temperature as the fluid bulk. Hence the phenomenon has local character and global character. Local as the exchange at any point depends on local object conditions, as well as a global character because incoming fluid near the object has been polluted by exchanges that have already occurred at many points along the boundary.

$\rho C u(r) \frac{\partial T}{\partial z}=k\left(\frac{\partial^{2} T}{\partial z^{2}}+\frac{1 \partial}{r \partial r}\left(r \frac{\partial T}{\partial r}\right)\right)$

A simple expression is desired by engineers for design purposes. This expression is usually cast in the form of the so-called Newton's law of cooling, which is can be formulated for internal convection as:

$q_{w}=h\left(T_{w}-T_{b}\right)$

Where, $q_{w}$ is the surface heat flux density entering the fluid domain, $T_{w}$ is the surface temperature at which heat is transferred, and $T_{b}$ is the bulk average temperature over a section normal to fluid flow, which satisfies the first law of thermodynamics. The so-called HTC is supposed to represent the complex relation in a simple form. Correlations for the HTC are only available in a very limited number of cases, which are:

- At duct wall, either uniform heat flux density or uniform temperature are imposed

- At duct inlet, either flat inlet temperature profile or fully developed temperature (i.e. same as outlet temperature)

The problem is that outside these very limited cases, no correlation exists. Moreover, all of these cases are difficult to realize in practice, even if a local HTC is defined, such as:

$q_{w}(x)=h(x) \Delta T(x)$

Above problems still persist as the obtained value is only applicable to the same limited set of problems. Although $h$ is more familiar and easy to be used, especially for hand calculations, it is too simple to capture the nature 
of convection physics. Hence, while $h$ is expected to give suitable results for standard (limited) cases, it may be very poor in other practical cases. In particular, for conjugate problems, at the solid / fluid interface neither $T_{w}$ nor $q_{w}$ are uniform.

Energy equation (Eq. (1)) contains both convection, that is global and diffusion, which is local. The former gives the system an elliptic character. The temperature difference between the object and the fluid at a point depends on all heat flux densities at all boundary points. Vice versa, the heat exchanged at a given surface point depends on temperatures at all boundary points. The convective term gives the problem a parabolic character, as there is a preferential direction related to downstreamupstream directions.

It has been proved [12] that the most general solution of energy equation can be cast in the following form:

$$
T\left(x^{\prime}\right)-T_{r e f}=\int_{\Omega} G\left(x, x^{\prime}\right) q_{v} d x+\int_{\partial \Omega} G\left(x, x^{\prime}\right) q_{S} d x
$$

Where $G\left(x, x^{\prime}\right)$ is a known kernel. It is unique and can be obtained analytically for simple geometries [13] and uniform velocity profile, $u$. Otherwise, it can always be obtained experimentally or numerically, but experiments would be quite involved. An arbitrary reference temperature is needed as we can only model temperature differences no single values.

No simplifying assumptions were made in order to obtain the integral form (Eq. (4)) starting from energy equation (Eq. (1)). Virtually, Eq. (1) can be recovered out of Eq. (4) through mathematical operations. This form plays a main role as it directly relates heat fluxes and temperatures at thermal ports. Out of which, a CTM can be deduced after making different simplifying assumptions. Both the local HTC as well as the special case of uniform HTC are special cases of Eq. (4), which were discussed in details [14].

\subsection{General Linear Compact Model}

In order to obtain a simple algebraic and linear form out of Eq. (1), it is needed to "discretize" it. Following the FP approach, this will be done by expressing both heat flux density $q$ and temperature $T$ over each thermal port i, by a truncated series. In fact, $q$ and $T$ can be expanded over any basis consisting of a complete set of known functions $\varphi_{i k}$ (for example, Fourier or Legendre series), without any loss of precision. The only approximation needed to discretize Eq. (4) is to truncate this series. The order of magnitude of the error due to discretization has been proven in [12].

$\left.T\right|_{x^{\prime} \in \Omega_{i}}-T_{r e f}=\sum_{k=1}^{K} T_{i k} \varphi_{i k}\left(x^{\prime}\right)$

$\left.q\right|_{x \in \Omega_{j}}=\sum_{l=1}^{L} q_{j l} \varphi_{j l}(x)$

Where $T_{i k}$ and $q_{j l}$ are expansion coefficients over the known series $\varphi_{i k}\left(x^{\prime}\right)$ as well as $\varphi_{j l}(x)$. The first index is the "port index", and the second one is the "mode index". A uniform $q$ or $T$ has the mode index 0 . For simplicity, the series is assumed to form an orthonormal set. Substituting this expansion in Eq. (4), multiplying by $\varphi_{i k}\left(x^{\prime}\right)$, and integrating with respect to $x^{\prime}$ give

$T_{i k}=\sum_{j=1}^{N} \sum_{l=1}^{L} R_{i k, j l} q_{j l}$

Where $R_{i k, j l}$ is a matrix of known constants defined by

$R_{i k, j l}=\int_{x^{\prime}} \int_{x} \varphi_{i k}\left(x^{\prime}\right) G\left(x, x^{\prime}\right) \varphi_{j l}(x) d x d x^{\prime}$

The proposed matrix form has two major advantages:

1. It can model any of the standard cases (uniform $T$ or uniform $q$ ), including other "nonstandard" ones, with a single matrix.

2. Conjugate convection becomes no more a problem. In fact, each domain (fluid and solid) will have its own matrix model similar to Eq. (8), which is constructed independently.

\subsection{Compact Model Solves Heat Transfer Coefficient Problems}

The simplicity of Newton's law of cooling (Eq. (2)) in modeling convection problem, should be an indication that this model has serious defects. This law is too simple to dissect a large number of convective heat transfer problems found in reality. Practical problems with this form were discussed [26] in order to realize its limitations and why the CTM can perform better. Hence, the CTM is considered to be widely used in modeling convection problems.

The purpose of this work is to obtain a more general simple expression for any heat flux density and temperature profiles using CTM which offers many advantages over the simple HTC approach (i.e. any space distribution, not just uniform).

\subsection{Geometry}

\section{MODEL DESCRIPTION}

A straight duct with a circular cross sectional area was used for the calculation as shown in Error! Reference source not found.

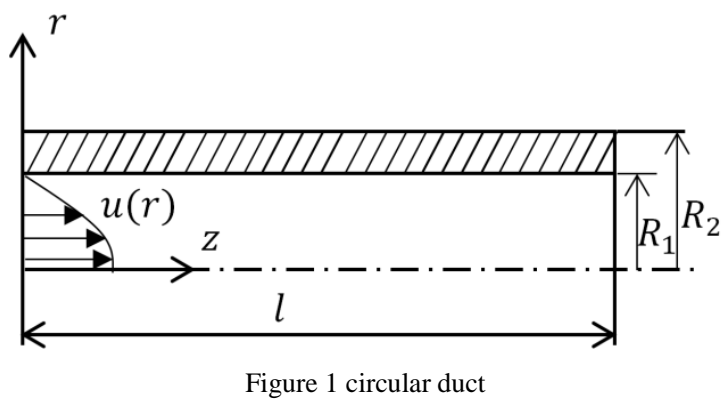

\subsection{List of Assumptions}

- Constant physical properties $\rho, \mu, k, C$

- Axisymmetric velocity and temperature field

- Negligible viscous dissipation 
- Hydrodinamically fully developed laminar flow

- The duct material is isotropic and homogeneous

\section{$2.3 \quad$ Governing Equations}

Based on the listed assumptions, governing equations can be written as follows;

\subsubsection{Convection Side}

Continuity, momentum and energy equations through the fluid can be written as:

$\frac{\partial u}{\partial z}+\frac{\partial v}{\partial r}+\frac{v}{r}=0$

$u \frac{\partial u}{\partial z}+v \frac{\partial u}{\partial r}=v\left(\frac{\partial^{2} u}{\partial r^{2}}+\frac{1}{r}\left(\frac{\partial u}{\partial r}\right)\right)$

$\rho C u(r) \frac{\partial T_{f}}{\partial z}=k_{f}\left(\frac{\partial^{2} T_{f}}{\partial z^{2}}+\frac{1 \partial}{r \partial r}\left(r \frac{\partial T_{f}}{\partial r}\right)\right)$

\subsubsection{Conduction Side}

Energy equation through the solid side can be formulated as;

$k_{S}\left(\frac{\partial^{2} T_{S}}{\partial z^{2}}+\frac{1 \partial}{r \partial r}\left(r \frac{\partial T_{S}}{\partial r}\right)\right)=0$

\subsection{Boundary Conditions}

\subsubsection{Convection:}

$q\left(R_{1}, z\right)=k_{f} \frac{\partial T_{f}}{\partial r}=q_{w}(z)$

$\mathrm{T}_{\mathrm{f}}(\mathrm{r}, 0)=\mathrm{T}_{\mathrm{in}}(\mathrm{r})$

$u(r)=2 U_{\text {ave }}\left(1-\frac{r^{2}}{R_{1}^{2}}\right)$

The applied inlet temperature and velocity, which are expressed by Eq. (14) and Eq. (15), programmed in C language and interpreted as User Defined Function (UDF) with ANSYS Fluent simulator.

\subsubsection{Conduction:}

$\frac{\partial T_{S}(r, 0)}{\partial r}=\frac{\partial T_{s}(r, l)}{\partial r}=0$

$k_{S} \frac{\partial T_{S}\left(R_{1}, z\right)}{\partial r}=q_{w 1}(z)$

$k_{s} \frac{\partial T_{s}\left(R_{2}, z\right)}{\partial r}=q_{w 2}(z)$

For conduction, all boundary conditions involve $\partial T_{s} / \partial r$. So, if $\boldsymbol{T}$ is a solution, $\boldsymbol{T}+C$ is also a solution (note that $C$ is an arbitrary constant). Thus, a reference temperature should be taken to get the right solution.

\subsubsection{Interface:}

$T_{S}\left(R_{1}, z\right)=T_{f}\left(R_{1}, z\right)$ $k_{s} \frac{\partial T_{s}\left(R_{1}, z\right)}{\partial r}=k_{f} \frac{\partial T_{f}\left(R_{1}, z\right)}{\partial r}$

\section{ANALYTIC SOLUTION FOR CONDUCTION}

Temperature distribution is developed over a series:

$T_{s}(r, z)=\sum_{n=0}^{\infty} \beta_{n}(r) \cos \left(\frac{n \pi z}{l}\right)$

Substituting in energy equation leads to:

$\frac{1 d}{r d r}\left(r \frac{d \beta_{n}}{\partial r}\right)-\alpha_{n}^{2} \beta_{n}=0$

where $\alpha=\frac{n \pi}{l}$

For $(n=0)$

$\beta_{n}(Y)=a_{0} \ln (r)+b_{0}$

For $(n \neq 0)$

$\beta_{n}(r)=a_{n} I_{0}\left(\alpha_{n} r\right)+b_{n} K_{0}\left(\alpha_{n} r\right)$

where $I, K$ are the modified Bessel functions of the first and the second orders.

Convection will be developed numerically using ANSYS Fluent.

\section{NUMERICAL SOLUTION FOR CONVECTION}

ANSYS Fluent [15] is one of the most-powerful CFD packages available, authorizing you to go faster and further as you optimize the performance of your product. The interactive solver setup, solution and post-processing capabilities of ANSYS Fluent make it easy to stop calculations, examine results, change any setting, and then continue the calculations within a single window.

The calculations were done for straight steel duct with thermal conductivity $k=16.27 \mathrm{~W} / \mathrm{m} . \mathrm{K}$. The dimensions of the duct with circular cross sectional area are listed below:

\begin{tabular}{l|l}
\multicolumn{2}{c}{ Table 1 dimensions of the circular duct } \\
Variable & Value \\
\hline Inner radius, $R_{1}$ & $0.0125 \mathrm{~m}$ \\
\hline Outer radius, $R_{2}$ & $0.0175 \mathrm{~m}$ \\
\hline Duct length, $l$ & $1.0 \mathrm{~m}$
\end{tabular}

Air is the used fluid and its properties [16] as listed in Table 2.

\begin{tabular}{l|l}
\multicolumn{2}{c}{ Table 2 properties of air } \\
\begin{tabular}{l|l} 
Variable & Value \\
\hline density, $\rho$ & $1.067 \mathrm{~kg} / \mathrm{m}^{3}$ \\
\hline Thermal conductivity, $k_{f}$ & $0.0285 \mathrm{~W} / \mathrm{m} . \mathrm{K}$ \\
\hline Specific heat, $c$ & $1009 \mathrm{~J} / \mathrm{kg} . \mathrm{K}$ \\
\hline Prandtl number, $P r$ & 0.71 \\
\hline Dynamic viscosity, $\mu$ & $2.01663 \mathrm{e}-5 \mathrm{~kg} / \mathrm{m} . \mathrm{K}$
\end{tabular}
\end{tabular}

The air enters the duct with an average velocity $U_{\text {ave }}=0.25 \mathrm{~m} / \mathrm{s}$. Thus, from the previous data the flow is considered to be laminar with Reynolds number, $R e=330$.

\subsection{Verification}

In order to perform a mesh independence study, some cases with different grid sizes were tested until an 
accepted error is reached. Due to the simple geometry of the problem, only one mesh type (rectangular) is tested.

\subsubsection{Mesh Dependency Study}

In this regard, four cases were examined for mesh dependency. The details of the four cases are listed in Table 3.

\begin{tabular}{c|c|c}
\multirow{2}{*}{ Tases } & Mesh type & $\begin{array}{c}\text { Cell size } \\
\mathrm{mm} / \text { cell }\end{array}$ \\
\hline Case 1 & Rectangular algorithm & 2 \\
\hline Case 2 & Rectangular algorithm & 1 \\
\hline Case 3 & Rectangular algorithm & 0.5 \\
\hline Case 4 & Rectangular algorithm & 0.25
\end{tabular}

The mass flow rate (m) was compared for the previous cases. Table 4 shows the corresponding mass flow rate and the relative error for each case.

\begin{tabular}{c|c|c} 
Table 4 Net mass flow rate for examined mesh dependency cases \\
Case & $\dot{m}, \mathrm{~kg} / \mathrm{h}$ & \% Error \\
\hline Case 1 & 0.4779 & 1.08 \\
\hline Case 2 & 0.4727 & 0.2 \\
\hline Case 3 & 0.4717 & 0.05 \\
\hline Case 4 & 0.4714 & --
\end{tabular}

Therefore, case 3 is selected for its accepted error and less running time.

\subsection{Validation}

Validation is made for convection only, as there is analytic solution for conduction. The present work (i.e. numerical solution) is compared to the analytical convection solution for standard cases. Two cases were validated, uniform heat flux $\left(q=100 \mathrm{w} / \mathrm{m}^{2}\right)$ and uniform wall temperature $(T=400 \mathrm{~K})$ with hydrodynamically developed flow for both cases. For constant heat flux, Figure 2 shows a comparison between analytical and numerical temperature distributions at different sections. The maximum relative error is $0.41 \%$.

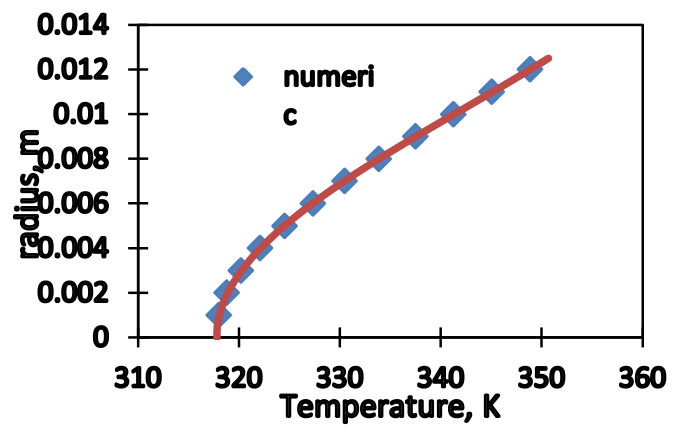

Figure 2 Analytical and numerical temperature distribution at $\mathrm{z}$ $=0.3 \mathrm{~m}$ for uniform $\mathrm{q}=100 \mathrm{~W} / \mathrm{m}^{2}$

Also, Figure 3 shows the differences between analytical and numerical temperature distributions at a radius of $0.0125 \mathrm{~m}$. The maximum relative error is $0.44 \%$.

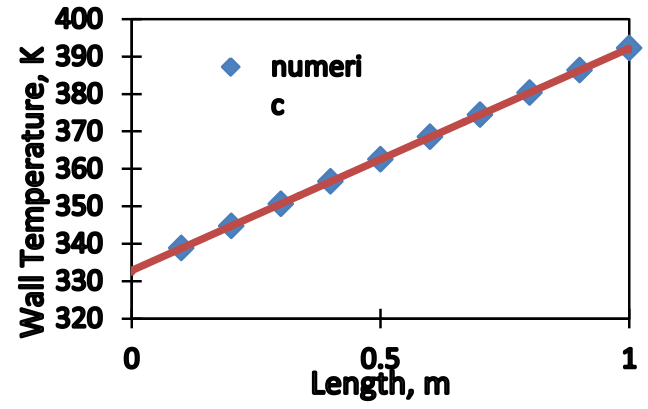

Figure 3 Analytical and numerical temperature distribution at $r$ $=0.0125 \mathrm{~m}$ and uniform $\mathrm{q}=100 \mathrm{w} / \mathrm{m}^{2}$

For uniform wall temperature, Figure 4 shows the differences between analytic and numerical temperature distributions at different sections. The maximum relative error is $0.65 \%$.

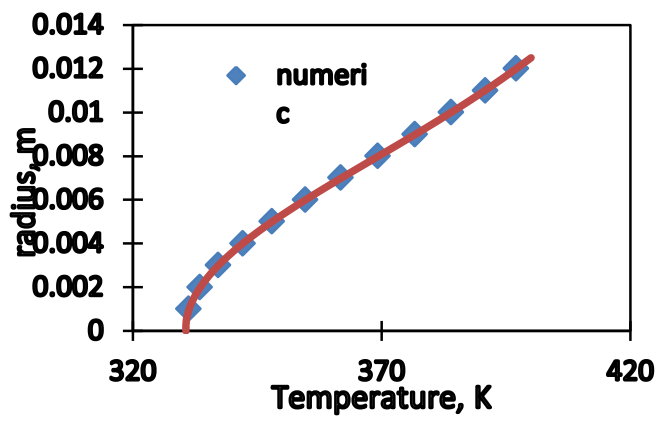

Figure 4 Analytical and numerical temperature distribution at $\mathrm{z}$ $=0.3 \mathrm{~m}$ for uniform wall temperature $\mathrm{T}=400 \mathrm{~K}$

Hence, the numerical solution is completely validated with the analytical one.

\subsection{Convection Model \\ 6.1.1 Constructing CTM}

In order to construct the matrix of CTM for convection side, various cases were solved numerically. This set listed below in Table 5. The inlet bulk temperature is $273 \mathrm{~K}$.

Table 5 various heat fluxes used to construct the convection matrix Heat flux, $q\left(\mathrm{~W} / \mathrm{m}^{2}\right)$

\begin{tabular}{l}
\hline$q=100$ \\
\hline$q=400 z$ \\
\hline$q=800 z^{2}$ \\
\hline$q=1400 z^{3}$ \\
\hline$q=1600 z^{4}$ \\
\hline$q=2000 z^{5}$ \\
\hline$q=2200 z^{6}$
\end{tabular}

Two inlet temperature profiles were simulated with zero heat flux at the inner duct wall. Table 6 lists these two profiles. 
Table 6 simulated inlet temperature profiles with zero heat flux Inlet temperature profile, $T(\mathrm{~K})$ $T=268+100000 r^{2}$

$T=269.7+80000000 r^{4}$

Thus, the whole CTM for convection is constructed.

\subsubsection{Testing CTM}

Three arbitrary cases were tested by the CTM to insure its validity.

In the first test, a uniform inlet temperature $(T=273 \mathrm{~K})$ and a variable heat flux $\left(q=80+190 z-300 z^{3}+\right.$ $\left.1500 z^{6} \mathrm{~W} / \mathrm{m}^{2}\right)$ at the wall were imposed.

Figure 5 shows the comparison between the CTM and numerical solution for the temperature distributions at the wall of the duct. Also, the maximum relative error is small $(0.96 \%)$.

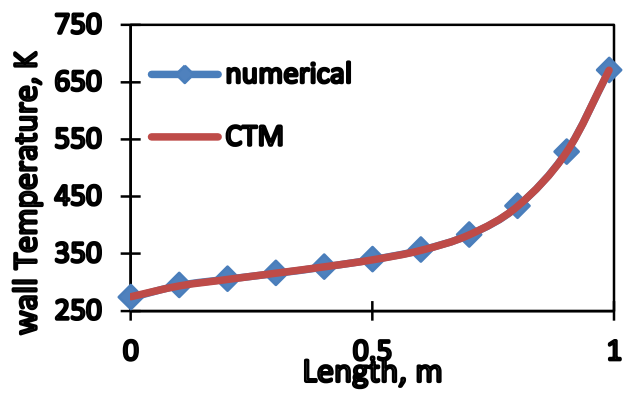

Figure 5 numerical and CTM solutions for temperature distribution at duct wall with uniform inlet temperature profile

Furthermore, in the second test, an inlet temperature profile $\left(T=267+115000 * r^{2} \mathrm{~K}\right)$ and a variable heat flux $\left(q=110+95 z-300 z^{4}+1500 z^{5} \mathrm{~W} / \mathrm{m}^{2}\right)$ at the wall were imposed.

Figure 6 shows the comparison between the CTM and numerical solution for the temperature distributions at the wall of the duct. The maximum relative error is $1.22 \%$.

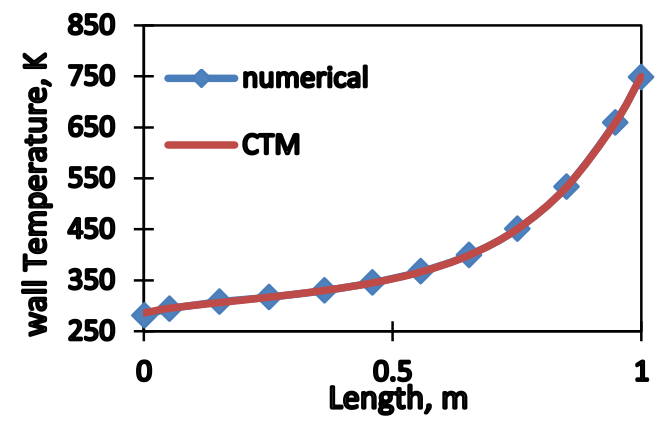

Figure 6 numerical and CTM solutions for temperature distribution at duct wall with parabolic inlet temperature profile
Finally, an inlet temperature profile $(T=268.4+$ $\left.95 E 7 * r^{4} \quad \mathrm{~K}\right)$ and a variable heat flux $(q=135-$ $250 z^{2}+320 z^{4}+900 z^{5} \mathrm{~W} / \mathrm{m}^{2}$ ) were imposed for the third test.

Figure 7 shows the comparison between the CTM and numerical solution for the temperature distributions at the wall of the duct. The maximum relative error is $2.7 \%$.

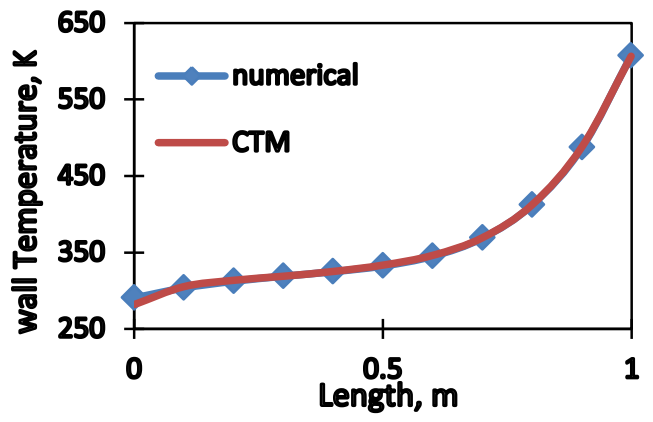

Figure 7 numerical and CTM solutions for temperature
distribution at duct wall with $4^{\text {th }}$ degree inlet temperature
profile

From the above results, it is obvious that the results of CTM agree well with that obtained from the numerical simulations. The error is greater at the first few centimeters of the duct as the heat conditions do not match together. Therefore, CTM is accepted for modeling convective heat transfer.

\subsection{Conjugate Heat Transfer}

Conjugate heat transfer problem was solved for a uniform heat flux at the outer surface of the circular duct $\left(q=80 \mathrm{~W} / \mathrm{m}^{2}\right)$ and an inlet temperature profile of $\left(T=270+473456.16 r^{2}-739847590.8 r^{4} \mathrm{~K}\right)$.

Conjugate problem was solved using CTM approach and compared with the numerical solution for the same boundary conditions.

Figure 8 and 9 illustrate the heat flux and the temperature distribution at the interface, respectively. It is shown that the differences between the temperature distribution results at the interface for CTM and numerical simulations are negligible. Also, the heat flux results obtained from the CTM concur with that of the numerical simulations. The differences between the heat flux results increases near the outlet due to the outlet effects and the truncated series of the analytic solution. 


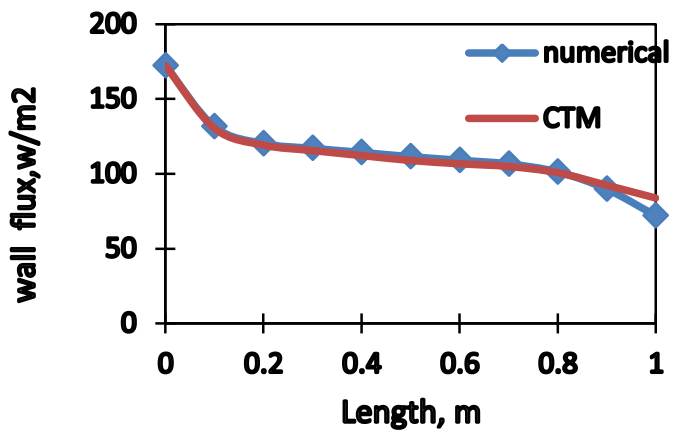

Figure 8 interface heat flux for CTM compared to numerical solution

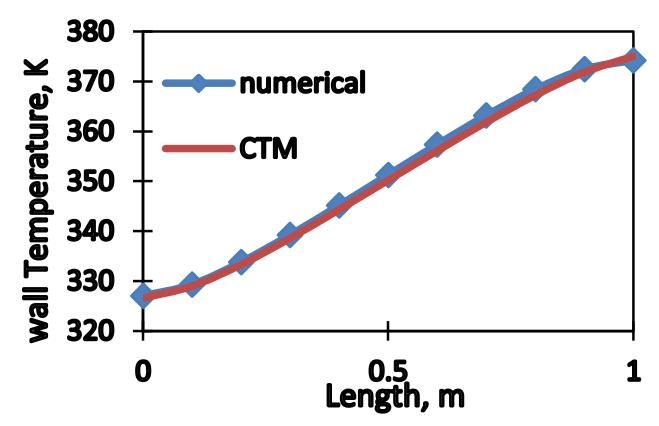

Figure 9 interface temperature for CTM compared to numerical solution

\section{CONCLUSION}

Application of the CTM approach for convection has been extended to cover

- Straight ducts with circular cross section area.

- Fully developed velocity profile at the duct inlet.

- Various inlet temperature profiles.

The advantages of the CTM also been observed which are

- The CTM can treat all standard cases (uniform T or $q$ ) as well as non-standard ones using the same model.

- The CTM takes into considerations the nonsymmetric nature of convective heat transfer.

- The CTM can easily treat the multiple heat source problems

The CTM gently treated the conjugate heat transfer by introducing a seamless merger of CTMs developed for solid domain and fluid domain independently. On the other hand, the HTC cannot treat conjugate heat transfer problems as we do not know which $q$ or $T$ profiles are present at the interface and it would not be possible to have many HTC correlations for any combination of fluid-solid domains.

\section{NOMENCLATURE}

\section{Roman Characters}

$\begin{array}{ll}C & \text { Specific heat J/kg.K } \\ h & \text { Heat transfer coefficient, W/m } \mathrm{m}^{2} \mathrm{~K} \\ k & \text { Thermal conductivity, W/m.K } \\ l & \text { Duct length, } \mathrm{m}\end{array}$

Mass flow rate, $\mathrm{kg} / \mathrm{h}$

Heat flux, W/m²

Radial coordinate

Inner duct radius, $\mathrm{m}$

Outer duct radius, $\mathrm{m}$

Temperature, $\mathrm{K}$

Average velocity, $\mathrm{m} / \mathrm{s}$

Arbitrary coordinate

Specified point on $x$ axis

Axial coordinate

\section{Greek Characters}

$\alpha \quad$ Used parameter in Eq. (22)

$\varphi \quad$ Basis function for expansion of

analytic solution

$\beta \quad$ Basis function for expansion of

conduction

$\mu \quad$ Dynamic viscosity, Pa.s

$v \quad$ Kinematic viscosity, $\mathrm{m}^{2} / \mathrm{s}$

$\rho \quad$ Density, $\mathrm{kg} / \mathrm{m}^{3}$

$\Omega \quad$ Problem domain

$\partial \Omega \quad$ Problem boundary

$\begin{array}{ll}\text { Subscripts } & \\ 1 & \text { Inner surface of the duct } \\ 2 & \text { Outer surface of the duct } \\ \text { ave } & \text { Average } \\ b & \text { Bulk } \\ f & \text { Fluid } \\ \text { in } & \text { Inlet } \\ \text { ref } & \text { Reference } \\ s & \text { Solid } \\ w & \text { Wall }\end{array}$

Acronyms

2D Two dimensional

3D Three dimensional

CFD Computational Fluid Dynamics

CTM Compact Thermal Model

FP Flexible profile

HTC Heat Transfer Coefficient

$\mathrm{Pe} \quad$ Peclet Number

Re Reynolds number

UDF User Defined Function

\section{REFERENCES}

[1.] Campo, A. and Schuler, C. "Heat transfer in laminar flow through circular tubes accounting for twodimensional wall conduction." International Journal of Heat and Mass Transfer 31.11 (1988): 2251-2259.

[2.] Aydin, O. Avci, M., Bali, T., and Arıcı, M. E. "Conjugate heat transfer in a duct with an axially varying heat flux." International Journal of Heat and Mass Transfer 76 (2014): 385-392.

[3.] Luna, N., F. Méndez, and Trevino, C. "Conjugated heat transfer in circular ducts with a power-law laminar convection fluid flow." International Journal of Heat and Mass Transfer 45.3 (2002): 655-666. 
[4.] Alzaharnah, I. T., M. S. Hashmi, and Yilbas, B. "Thermal stresses in thick-walled pipes subjected to fully developed laminar flow." Journal of Materials Processing Technology 118.1 (2001): 50-57.

[5.] Al-Zaharnah, I. T., B. S. Yilbas, and Hashmi, M. S. J. "Conjugate heat transfer in fully developed laminar pipe flow and thermally induced stresses." Computer Methods in Applied Mechanics and Engineering 190.8 (2000): 1091-1104.

[6.] Barozzi, G. S., and Pagliarini, G. "A method to solve conjugate heat transfer problems: the case of fully developed laminar flow in a pipe." Journal of Heat Transfer 107.1 (1985): 77-83.

[7.] Shah, K. and Jain, A. "An iterative, analytical method for solving conjugate heat transfer problems." International Journal of Heat and Mass Transfer 90 (2015): 1232-1240.

[8.] Luikov, A. V., Aleksashenko, V. A., and Aleksashenko, A. A. "Analytical methods of solution of conjugated problems in convective heat transfer." International Journal of Heat and Mass Transfer 14.8 (1971): 1047-1056.

[9.] Sabry, M.N. "Static and dynamic thermal modeling of ICs." Microelectronics Journal 30.11 (1999): 1085-1091.

[10.] Noebauer, G. "Creating compact models using standard spreadsheet software." Semiconductor
Thermal Measurement and Management, 2001. Seventeenth Annual IEEE Symposium. IEEE, 2001.

[11.] Sabry, M.N. "Compact thermal models for internal convection." Components and Packaging Technologies, IEEE Transactions on 28.1 (2005): 58-64.

[12.] Sabry, M.N. "Compact thermal models for electronic systems." Components and Packaging Technologies, IEEE Transactions on 26.1 (2003): 179-185.

[13.] Morse, P. M. and Feshbach, H. "Methods of theoretical physics." International Series in Pure and Applied Physics, New York: McGraw-Hill, 19531 (1953).

[14.] Sabry, M.N. "Generalization of the heat transfer coefficient concept for system simulation." Journal of Heat Transfer 133.6 (2011): 060905.

[15.] "ANSYS Fluent 15 Capabilities Brochure." Available:

http://www.ansys.com/Products/Simulation +Technology/Fluid+Dynamics/Fluid+Dyna mics+Products/ANSYS+Fluent

[16.] "Thermodynamic properties of air" Available: http://www.engineeringtoolbox.com/air-propertiesd_156.html 